\title{
Matrix Metalloproteinase-11 Gene Polymorphisms as a Risk for Hepatocellular Carcinoma Development in Egyptian Patients
}

\author{
Hind Saad ${ }^{1,2}$, Magdy A-H Zahran³, Olfat Hendy², Mohamed Abdel-Samiee ${ }^{4}$, \\ Hanan M Bedair², Eman Abdelsameea ${ }^{4 *}$
}

\begin{abstract}
Background: Chronic hepatitis $\mathrm{C}(\mathrm{CHC})$ virus infection is one of major risk factors of hepatocellular carcinoma (HCC) in Egypt, which is a major cause of cancer mortalityin the world. Matrix metalloproteinase-11 (MMP-11) has an important role in tumor migration and metastasis. Therefore, this study aimed to determine relation between $M M P-11$ gene polymorphisms and risk of HCC development among Egyptian cirrhotic patients. Subjects and methods: Two hundred and sixty patients were included, 140 of them with $\mathrm{HCC}$ on top of $\mathrm{CHC}$ and 120 patients with post CHC liver cirrhosis (LC) as well as 140 subjects were enrolled in the study as healthy controls. Two single nucleotide polymorphisms (SNPs) rs738791 and rs738792 for MMP-11 gene were done using real-time PCR. Results: Combination of CT and TT allele of rs738791 genotypes was more significantly frequent in HCC compared to LC patients and controls, however, a higher frequency of T allele was found in HCC patients compared to LC and controls. In spite of lake of significant difference between patient groups regarding the rs 738792 genotypes, the CC genotype was considered a risk of developing portal vein thrombosis, and was associated with advanced tumor stage, increased tumor size, higher Cancer of the Liver Italian Program [CLIP] score, more advanced Barcelona stage [D] and with child Pugh class [C]. Conclusion: Genetic variations in MMP-11 may be implicated in post HCV-HCC development and might be dependable biomarkers for HCC progression.
\end{abstract}

Keywords: Hepatocellular carcinoma- hepatitis C virus- single nucleotide polymorphism- MMP-11 polymorphisms

Asian Pac J Cancer Prev, 21 (12), 3725-3734

\section{Introduction}

Hepatocellular carcinoma (HCC) is one of cancer causes of death all over the world (Gupta et al., 2019). HCC follows frequent diverse causes of damage to the liver such as chronic hepatitis $\mathrm{C}$ and $\mathrm{B}$ infection, chronic use of alcohol and nonalcoholic fatty liver disease (EASL guidelines, 2018). Chronic HCV infection represented 75 to $85 \%$ of infected persons. Nearly, $20 \%$ of them develop complications which include cirrhosis or HCC after 20 years of occurrence of infection (Josep et al., 2019).

Matrix metalloproteinases (MMPs) have an essential role in biological behaviors of most solid malignant tumors. MMPs include a family of endopeptidases which has the capacity of extracellular matrix proteins degradation and remodeling. They have important roles for progression of invasion and also metastasis of malignant solid tumors (Kessenbrock et al., 2010). MMP-11 is a member of MMPs family, also known astromelysin-3, is one of stromelysin subgroup that belongs to MMP superfamily (Zhang et al., 2016). It has been associated with HCC and its expression controls miR-125a-regulated proliferation, epithelial-mesenchymal transition and metastasis of HCC (Geervliet and Bansal, 2020).

Genetic polymorphisms of MMP-11 have been found in different cancer types (Lin et al., 2015; Koleck et al., 2017). MMP-11 expression has been upregulated in human carcinomas as ovarian, breast, colorectal, lung and HCC (Rouyer et al., 1994; Bi et al., 2012) and minimal studies discussed its role in HCC.

Serum alfa fetoprotein (AFP) is the most common biomarker used in early $\mathrm{HCC}$ detection and prognostic factor for patient's survival and/ or recurrence of tumor (Chaiteerakij et al., 2015). However, lake of its specificity and low sensitivity for HCC detection (Zhu et al., 2013), the search about new markers with more sensitivity and specificity is needed. Therefore the current case-control study was designed to determine the relation between MM-11 genotypes and risk of HCC development among Egyptian cirrhotic patients and to find its relation to HCC

${ }^{1}$ Biochemistry Division, Department of Chemistry, Faculty of Science, Menoufia University, Shebein El-Kom, Egypt. ${ }^{2}$ Departement of Clinical Pathology, National Liver Institute, Menoufia University, Shebein El-Kom, Egypt. ${ }^{3}$ Chemistry Department, Faculty of Science, Menoufia University, Shebein El-Kom, Egypt. ${ }^{4}$ Departement of Hepatology and Gastroenterology, National Liver Institute, Menoufia University, Shebein El-Kom,Egypt.*For Correspondence:dreman555@yahoo.co.uk 
status.

\section{Materials and Methods}

All participants provided written informed consent prior starting in this study. The current study included: 260 patients were included, 140 of them with HCC on top of $\mathrm{CHC}$ and 120 patients with post $\mathrm{CHC}$ liver cirrhosis (LC). They were selected from the outpatient clinics of hepatology and gastroenterology department of the National Liver Institute (NLI), Menoufia University. HCC was diagnosed by the characteristic vascular enhancement pattern detected by multislice triphasic spiral CT scan and/ or MRI according to established diagnostic criteria (EASL guidelines, 2018).

Clinical HCC classification was based on TNM and Barcelona- Clinic Liver Cancer (BCLC) staging systems. Diagnosis of cirrhosis was based on clinical findings, imaging studies (abdominal ultrasound) and laboratory results. Severity of cirrhosis was graded according to Child Pugh classification (Pugh et al., 1973). Additionally, 140 subjects with gender and age matched to patients were enrolled in the study as healthy controls.

Exclusion criteria: Patients with other causes of chronic liver disease, patients with other liver tumors (e.g. adenoma), HCC cases on top of co-infection with Hepatitis $\mathrm{C}$ and $\mathrm{B}$, patients aged $<18$ years old, cases with chronic inflammatory diseases or autoimmune diseases were excluded from the study. Hematological malignancy and tumors of any organ other than the liver were of the exclusion criteria from the study.

The study was performed according to ethical standard of the responsible Institutional Committee and with the Helsinki Declaration of 1975 as revised in 2013. Either verbal or written consents were obtained from all subjects prior to enrollment in the study and the conventional data was collected from the patients' files. The study protocol was in accordance with declaration of Helsinki and approval of the Scientific and Ethical Committee of NLI, Menoufia University.

\section{Laboratory investigations}

Ten $\mathrm{ml}$ of venous blood were drawn from all subjects included in this study by venipuncture from were divided into: $2 \mathrm{ml}$ was put in vacutainer tubes containing EDTA for molecular testing of polymorphism by real time PCR; $2 \mathrm{ml}$ was put in EDTA tube for complete blood count (CBC) and the $1.8 \mathrm{ml}$ was put in tube contains $0.2 \mathrm{ml}$ of sodium citrate $3.8 \%$ for coagulation screening profile. The remaining volume was collected in plain vacutainer and allowed to clot and serum separated for liver function tests as alanine aminotransferase (ALT), aspartate aminotransferase (AST), albumin, total and direct bilirubin, hepatitis virus markers and AFP level.

CBC was done using Sysmex XT-1800i automated hematology analyzer (Sysmex, Japan), liver functions tests using Cobas e501 Auto analyzer (Roche, Germany), and AFP measurement using enzyme immunoassay based on electro-chemiluminescence immunoassay by Cobas e601 Auto analyzer (Roche). Prothrombin time (PT) and international normalized ratio (INR) value was done by
BFT II fibrintimer. HBsAg and HCV antibodies were done by the ectroche-miluminescence immunoassay "ECLIA" using Cobas 6000 (e601 module).

\section{Genotyping of MMP-11}

Genomic DNA was extracted from EDTA samples using QIAamp DNA Mini Kit (catalog number, 51106, Qiagen, Santa Clarita, USA). MMP-11 was genotyped by real-time polymerase chain reaction (PCR) using fluorescent labeled probes. Single nucleotide polymorphism (SNP) was done for two MMP-11 variants (rs738791 and rs738792) as previously reported (Tecnologies, 2013). The ABI TaqMan allelic discrimination kit (catalog number 4351379, Applied Biosystems, Carlsbad, CA), briefly, the fluorescent labeled probes: GCCAGTGGTGGCCTGTGTCTACCAC[C/T] TCACCTCACTGAACCTGAGAGTCC (VIC dye for allele C, FAM for allele T) for rs738791. The probes: CATCCTCCTGCCTAGGACG[C/T] CCACCACCTCCATGCCGAGAGGAGG (VIC dye for allele T, FAM dye for allele C) was fluorescent probes for rs 738792. The PCR fluorescence product was detected on a Rotor-Gene Q System (QIAGEN, GmbH- Germany) and the intensity of the fluorescence signal was analyzed by Rotor-Gene Q series software. The reaction was performed in a 96-well format in a total reaction volume of $25 \mu \mathrm{l}$ using $20 \mathrm{ng}$ of genomic DNA. The reaction was heated for $2 \mathrm{~min}$ at $50^{\circ} \mathrm{C}$, then $10 \mathrm{~min}$ at $95^{\circ} \mathrm{C}$, followed by 40 cycles of $95^{\circ} \mathrm{C}$ for $15 \mathrm{sec}$ and $60^{\circ} \mathrm{C}$ for $1.5 \mathrm{~min}$. The rs738791 and rs738792 genotyping results were recognized consequently by allele-separation dependent on relative enhancement of every particular fluorophore, adjusted for control Rox fluorophore.

\section{Statistical analysis}

Data were fed to the computer and analyzed using IBM SPSS software package version 22.0. (Armonk, NY: IBM Corp). Quantitative data were described mean, standard deviation (SD) or median and interquartile range (IQR). Chi-square test was used for categorical variables to compare between different groups. Mann Whitney test for abnormally distributed quantitative variables to compare between two studied groups. Kruskal Wallis test for abnormally distributed quantitative variables, to compare between more than two studied groups. Post Hoc for pairwise comparisons. Significance of the obtained results was judged at the level below 5\%. Odd ratio (OR and $95 \%$ Confidence Interval of an event occurring in one risk group was calculated to the odds of it occurring in the non-risk group. The population of the studied sample was explored to find its equilibrium with Hardy-Weinberg equation by Hardy-Weinberg and regression to detect the most independent/ affecting factor for affecting HCC.

\section{Results}

The gender and age were matched among the studied groups. DM was significantly more prevalent among patients with LC $(\mathrm{p}=0.032)$. There was a significant increase among studied groups as regards ALT, AST, total bilirubin and AFP compared to healthy control, while as regards serum albumin level and platelets among 
the studied groups, they were significantly decreased (p-value $<0.001$ for each). No significant difference was detected between HCC and LC groups regarding smoking $(\mathrm{p}=0.407)$. Portal vein thrombosis is more frequent in HCC more than LC group $(\mathrm{p}<0.001)$; while no significant
Matrix Metalloproteinase-11 Gene Polymorphisms in HCC difference was found between HCC and liver cirrhosis patients regarding Child -Pugh score $(\mathrm{p}=0.073)$ (Table 1).

No significant difference among studied groups was found regards the SNP rs738791 genotypes $(p=0.171)$. While the CT and TT genotypes combination was more

Table 1. Comparison of Demographic and Laboratory Data among the 3 Studied Groups

\begin{tabular}{|c|c|c|c|c|c|c|}
\hline \multirow[t]{2}{*}{ Parameters } & \multicolumn{2}{|c|}{$\mathrm{HCC}(\mathrm{n}=140)$} & \multicolumn{2}{|c|}{$\mathrm{LC}(\mathrm{n}=120)$} & Controls $(n=140)$ & \multirow[t]{2}{*}{$\mathrm{p}$ - value } \\
\hline & No. & $\%$ & No. & $\%$ & No. & \\
\hline \multicolumn{7}{|l|}{ Gender } \\
\hline Male & 107 & 76.4 & 78 & 65 & 73.6 & 0.108 \\
\hline Female & 33 & 23.6 & 42 & 35 & 26.4 & \\
\hline \multicolumn{7}{|c|}{$\mathrm{p}_{1}=0.054, \mathrm{p}_{2}=0.581, \mathrm{p}_{3}=0.134$} \\
\hline \multicolumn{7}{|l|}{ Age (years) } \\
\hline Range & $43.0-66.0$ & & \multicolumn{2}{|c|}{$42.0-70.0$} & $55.0-70.0$ & 0.343 \\
\hline \multirow[t]{2}{*}{ Mean $\pm \mathrm{SD}$} & $59.47 \pm 4.72$ & & \multicolumn{2}{|c|}{$58.85 \pm 6.64$} & \multirow[t]{2}{*}{$59.34 \pm 3.58$} & \\
\hline & & & $943, \mathrm{p}$ & $08, \mathrm{p}_{3}=0.418$ & & \\
\hline \multicolumn{7}{|l|}{$\mathrm{DM}$} \\
\hline No & 84 & $60.00 \%$ & 56 & $46.70 \%$ & & $0.032 *$ \\
\hline Yes & 56 & $40.00 \%$ & 64 & $53.30 \%$ & & \\
\hline \multicolumn{7}{|l|}{ Smoking } \\
\hline No smokers & 84 & $60.00 \%$ & 78 & $65.00 \%$ & & 0.407 \\
\hline Smokers & 56 & $40.00 \%$ & 42 & $35.00 \%$ & & \\
\hline \multicolumn{7}{|l|}{$\operatorname{ALT}(\mathrm{U} / \mathrm{L})$} \\
\hline Mean \pm SD & \multicolumn{2}{|c|}{$54.70 \pm 44.45$} & \multicolumn{2}{|c|}{$38.20 \pm 18.33$} & \multirow[t]{2}{*}{$22.69 \pm 4.06$} & $<0.001^{*}$ \\
\hline & & & $13^{*}, \mathrm{p}_{2}$ & $1^{*}, \mathrm{p}_{3}<0.001^{*}$ & & \\
\hline \multicolumn{7}{|l|}{ AST (U/L) } \\
\hline \multirow[t]{2}{*}{ Mean \pm SD } & \multirow{2}{*}{\multicolumn{2}{|c|}{$82.07 \pm 78.24$}} & \multicolumn{2}{|c|}{$63.33 \pm 63.28$} & \multirow[t]{2}{*}{$19.74 \pm 4.73$} & $<0.001^{*}$ \\
\hline & & & $21, \mathrm{p}_{2}$ & $1^{*}, \mathrm{p}_{3}<0.001^{*}$ & & \\
\hline \multicolumn{7}{|l|}{ Albumin $(\mathrm{g} / \mathrm{dl})$} \\
\hline Mean \pm SD & $2.78 \pm 0.88$ & & & 0.76 & $4.47 \pm 0.22$ & $<0.001 *$ \\
\hline & & & $66, \mathrm{p}_{2}$ & $1^{*}, \mathrm{p}_{3}<0.001^{*}$ & & \\
\hline Total bilirubin ( $\mathrm{m}$ & & & & & & \\
\hline Median (IQR) & $1.55(1.10$ & $5.80)$ & 1.85 & $-4.02)$ & $0.42(0.36-0.60)$ & \\
\hline & & & $30, \mathrm{p}_{2}$ & $1^{*}, \mathrm{p}_{3}<0.001^{*}$ & & \\
\hline Platelets $\left(\times 10^{3} / \mathrm{ml}\right.$ & & & & & & \\
\hline Mean \pm SD & $132.8 \pm 90.47$ & & & 83.65 & $290.9 \pm 67.41$ & $<0.001^{*}$ \\
\hline & & & $67, \mathrm{p}_{2}$ & $1^{*}, \mathrm{p}_{3}<0.001^{*}$ & & \\
\hline $\operatorname{AFP}(n g / d l)$ & & & & & & $<0.001^{*}$ \\
\hline Median (IQR) & $152.25(6.6$ & $-1400)$ & 2.5 & $-5.4)$ & $2.10(1.9-2.9)$ & \\
\hline & & & $01^{*}, \mathrm{p}_{2}$ & $1^{*}, \mathrm{p}_{3}=0.028^{*}$ & & \\
\hline & $\mathrm{N}$ & $\%$ & $\mathrm{~N}$ & $\%$ & & \\
\hline PVT & & & 112 & 93.3 & & \\
\hline No & 82 & 58.6 & 8 & 6.7 & & $<0.001^{*}$ \\
\hline Yes & 58 & 41.4 & & & & \\
\hline Child Pugh class & & & & & & \\
\hline $\mathrm{A}$ & 48 & 34.3 & 26 & 21.7 & & \\
\hline $\mathrm{B}$ & 52 & 37.1 & 56 & 46.7 & & \\
\hline $\mathrm{C}$ & 40 & 28.6 & 38 & 31.7 & & \\
\hline
\end{tabular}

$\chi^{2}$, Chi square test; H, H for Kruskal Wallis test; MC, Monte Carlo; SD, Standard deviation; HCC, hepatocellular carcinoma; LC, liver cirrhosis; DM, Diabetes mellitus; ALT, alanine aminotransferase; AST, aspartate aminotransferase; AFP, Alpha fetoprotein; $\mathrm{p}_{1}$, value for comparing between HCC and liver cirrhosis; $\mathrm{p}_{2}$, value for comparing between HCC and control; p3, value for comparing between liver cirrhosis and control;*, Statistically significant at $\mathrm{p}<0.05$ 
Table 2. Comparison of Genotypes and Allele Frequencies of MMP-11 (rs738791 and rs738792) among the 3 Studied Groups

\begin{tabular}{|c|c|c|c|c|c|c|c|}
\hline \multirow[t]{2}{*}{ Variable } & \multicolumn{2}{|c|}{$\operatorname{HCC}(\mathrm{n}=140)$} & \multicolumn{2}{|c|}{$\mathrm{LC}(\mathrm{n}=120)$} & \multicolumn{2}{|c|}{ Controls $(n=140)$} & \multirow[t]{2}{*}{$\mathrm{p}$-value } \\
\hline & No. & $\%$ & No. & $\%$ & No. & $\%$ & \\
\hline \multicolumn{8}{|c|}{ rs738791 Genotype } \\
\hline $\mathrm{CC}$ & 52 & 37.1 & 50 & 41.7 & 72 & 51.4 & 0.171 \\
\hline $\mathrm{CT}$ & 70 & 50 & 58 & 48.3 & 56 & 40 & \\
\hline $\mathrm{TT}$ & 18 & 12.9 & 12 & 10.0 & 12 & 8.6 & \\
\hline $\mathrm{CT}+\mathrm{TT}$ & 88 & 62.9 & 70 & 58.3 & 68 & 48.6 & $0.049 *$ \\
\hline \multicolumn{8}{|c|}{ Allele frequency } \\
\hline $\mathrm{C}$ & 174 & 62.1 & 158 & 65.8 & 200 & 71.4 & 0.064 \\
\hline $\mathrm{T}$ & 106 & 37.9 & 82 & 34.2 & 80 & 28.6 & \\
\hline \multicolumn{8}{|c|}{ rs738792 Genotype: } \\
\hline $\mathrm{TT}$ & 66 & 47.1 & 60 & 50.0 & 70 & 50 & 0.901 \\
\hline $\mathrm{TC}$ & 62 & 44.3 & 48 & 40.0 & 60 & 42.9 & \\
\hline $\mathrm{CC}$ & 12 & 8.6 & 12 & 10.0 & 10 & 7.1 & \\
\hline $\mathrm{TC}+\mathrm{CC}$ & 74 & 52.9 & 60 & 50.0 & 70 & 50 & 0.862 \\
\hline \multicolumn{8}{|c|}{ Allele frequency } \\
\hline $\mathrm{T}$ & 194 & 69.3 & 168 & 70.0 & 200 & 71.4 & 0.853 \\
\hline $\mathrm{C}$ & 86 & 30.7 & 72 & 30.0 & 80 & 28.6 & \\
\hline
\end{tabular}

$\chi^{2}$, Chi square test; SNP, Single nucleotide polymorphism; $\mathrm{p}, \mathrm{p}$ value for comparing between the 3 studied groups; ${ }^{*}$, Statistically significant at $\mathrm{p}$ $<0.05$.

frequent in HCC group compared to LC and control $(p=0.049)$. Also, a higher frequency of $\mathrm{T}$ allele was detected in HCC patients (37.9\%) compared to cirrhotic patients $(34.2 \%)$ and healthy controls $(28.6 \%)$ without statistical significant difference (Table 2).

No significant difference was observed among studied groups as regards SNP rs738792 genotype (TT, TC or $\mathrm{CC})$, allele distributions ( $\mathrm{T}$ or $\mathrm{C})(\mathrm{p}=0.901$ and 0.853 respectively) or combination of $\mathrm{TC}+\mathrm{CC}$ genotypes $(\mathrm{p}=0.712)($ Table 2$)$.

The risk of hepatocellular carcinoma among different genotypes of rs 738791 (TT and CT genotype) was significantly increased by 2.077 fold $(\mathrm{p} 1=0.018)$ and 1.731 fold $(\mathrm{p} 1=0.032)$ respectively when compared to healthy control. Additionally, the patients carrying heterozygous (CT+TT) genotype and T allele had a significant increased 1.791 fold $(\mathrm{p} 1=0.016)$ and 1.523 fold $(\mathrm{p} 1=0.020)$ respectively when compared to controls.

Table 3. The Odds Ratios among the Three Studied Groups According to rs738791 and rs738792 Genotypes

\begin{tabular}{|c|c|c|c|c|c|c|}
\hline Variable & $\mathrm{p}_{1}$ & OR1 (CI. 95\%) & $\mathrm{p}_{2}$ & OR2 (CI. 95\%) & $\mathrm{p}_{3}$ & OR3 (CI. 95\%) \\
\hline \multicolumn{7}{|c|}{ rs738791 Genotype } \\
\hline $\mathrm{CC}^{\circledR}$ & 1 & & & 1 & & 1 \\
\hline $\mathrm{CT}$ & $0.032 *$ & $1.731(1.049-2.856)$ & 0.128 & $1.491(0.891-2.496)$ & 0.576 & $1.161(0.69-1.95)$ \\
\hline TT & 0.018 & $2.077(0.921-4.682)$ & 0.416 & $1.440(0.599-3.464)$ & 0.386 & $1.442(0.63-3.30)$ \\
\hline $\mathrm{CT}+\mathrm{TT}$ & $0.016^{*}$ & $1.791(1.11-2.89)$ & 0.116 & $1.482(0.91-2.42)$ & 0.457 & $1.219(0.73-1.99)$ \\
\hline \multicolumn{7}{|c|}{ Allele frequency: } \\
\hline $\mathrm{C}^{\circledR}$ & 1 & & & & & 1 \\
\hline $\mathrm{T}$ & $0.020^{*}$ & $1.523(1.069-2.171)$ & 0.17 & $1.298(0.894-1.882)$ & 0.383 & $1.738(0.82-1.68)$ \\
\hline \multicolumn{7}{|c|}{ rs738792 Genotype } \\
\hline $\mathrm{TT}^{\circledR}$ & 1 & & & 1 & & 1 \\
\hline $\mathrm{TC}$ & 0.713 & $1.096(0.672-1.787)$ & 0.792 & $0.933(0.559-1.559)$ & 0.54 & $1.174(0.70-1.96)$ \\
\hline $\mathrm{CC}$ & 0.601 & $1.273(0.515-3.143)$ & 0.467 & $1.400(0.565-3.469)$ & 0.831 & $0.909(0.38-2.18)$ \\
\hline $\mathrm{TC}+\mathrm{CC}$ & 0.632 & $0.892(0.56-1.43)$ & 1 & $1.0(0.61-1.62)$ & 0.646 & $0.892(0.55-1.45)$ \\
\hline \multicolumn{7}{|c|}{ Allele frequency: } \\
\hline $\mathrm{T}^{\circledR}$ & 1 & & & & & 1 \\
\hline $\mathrm{C}$ & 0.579 & $1.108(0.771-1.593)$ & 0.721 & $1.071(0.734-1.565)$ & 0.86 & $1.034(0.71-1.50)$ \\
\hline
\end{tabular}

CI, Confidence interval; OR1, Odds ratio for HCC and Control; $\mathrm{P}_{1}$, Comparison between HCC and Control; OR, Odds ratio for LC and Control; $\mathrm{P}_{2}$, Comparison between LC and control; $\mathrm{OR}_{3}$, Odds ratio for HCC and LC; $\mathrm{P}_{3}$, Comparison between HCC and LC; $\mathrm{p}^{*}$, Statistically significant at $\mathrm{p}<0.05$ 


$$
\mathrm{CC}(\mathrm{n}=52) \quad \mathrm{CT}(\mathrm{n}=70) \quad \mathrm{TT}(\mathrm{n}=18)
$$

\begin{tabular}{|c|c|c|c|c|c|c|c|}
\hline & No. & $\%$ & No. & $\%$ & No. & $\%$ & \\
\hline \multicolumn{8}{|l|}{ Sex } \\
\hline Male & 41 & 78.8 & 52 & 74.3 & 14 & 77.8 & 0.833 \\
\hline Female & 11 & 21.1 & 18 & 25.7 & 4 & 22.2 & \\
\hline \multicolumn{8}{|l|}{ Age (years) } \\
\hline Range & $43.0-65.0$ & & $50.0-66.0$ & & $55.0-65.0$ & & 0.747 \\
\hline Mean \pm SD & $58.96 \pm 5.28$ & & $59.60 \pm 4.48$ & & $60.44 \pm 3.94$ & & \\
\hline \multicolumn{8}{|l|}{ Lymph nodes } \\
\hline No & 44 & 84.6 & 58 & 82.9 & 18 & 100 & 0.172 \\
\hline Yes & 8 & 15.4 & 12 & 17.1 & 0 & 0 & \\
\hline \multicolumn{8}{|l|}{ Metastasis } \\
\hline No & 52 & 100 & 66 & 94.3 & 18 & 100 & $\mathrm{MCp}=$ \\
\hline Yes & 0 & 0 & 4 & 5.7 & 0 & 0 & 0.184 \\
\hline \multicolumn{8}{|l|}{$\mathrm{T}$ (Tumor stage) } \\
\hline $\mathrm{T} 1$ & 22 & 42.3 & 22 & 31.4 & 4 & 22.2 & $\mathrm{MCp}=$ \\
\hline $\mathrm{T} 2$ & 24 & 46.2 & 26 & 37.1 & 6 & 33.3 & 0.056 \\
\hline $\mathrm{T} 3$ & 6 & 11.5 & 20 & 28.6 & 8 & 44.4 & \\
\hline $\mathrm{T} 4$ & 0 & 0 & 2 & 2.9 & 0 & 0 & \\
\hline \multicolumn{8}{|l|}{ PVT } \\
\hline No & 38 & 73.1 & 38 & 54.3 & 6 & 33.3 & $0.008 *$ \\
\hline Yes & 14 & 26.9 & 32 & 45.7 & 12 & 66.7 & \\
\hline \multicolumn{8}{|l|}{$\operatorname{AFP}(\mathrm{ng} / \mathrm{ml})$} \\
\hline Median & 41.79 & & 287 & & 405.2 & & 0.097 \\
\hline Focal lesion size & & & & & & & 0.073 \\
\hline$<3 \mathrm{~cm}$ & 22 & 42.3 & 14 & 20 & 4 & 22.2 & \\
\hline$>3 \mathrm{~cm}$ & 30 & 57.7 & 56 & 80 & 14 & 77.8 & \\
\hline \multicolumn{8}{|c|}{ CLIP score for HCC } \\
\hline 0 & 10 & 19.2 & 2 & 2.9 & 0 & 0 & $\mathrm{MCp}$ \\
\hline 1 & 14 & 26.9 & 10 & 14.3 & 0 & 0 & $<0.001 *$ \\
\hline 2 & 10 & 19.2 & 20 & 28.6 & 2 & 11.1 & \\
\hline 3 & 8 & 15.4 & 14 & 20 & 4 & 22.2 & \\
\hline 4 & 8 & 15.4 & 12 & 17.1 & 4 & 22.2 & \\
\hline 5 & 2 & 3.8 & 6 & 8.6 & 8 & 44.4 & \\
\hline 6 & 0 & 0 & 6 & 8.6 & 0 & 0 & \\
\hline \multicolumn{8}{|c|}{ BCLC score for HCC } \\
\hline A & 14 & 26.9 & 10 & 14.3 & 0 & 0 & $<0.001 *$ \\
\hline $\mathrm{B}$ & 22 & 42.3 & 16 & 22.9 & 2 & 11.1 & \\
\hline $\mathrm{C}$ & 10 & 19.2 & 20 & 28.6 & 6 & 33.3 & \\
\hline $\mathrm{D}$ & 6 & 11.5 & 24 & 34.3 & 10 & 55.6 & \\
\hline \multicolumn{8}{|l|}{ Child score } \\
\hline A & 24 & 46.2 & 24 & 34.3 & 0 & 0 & $<0.001 *$ \\
\hline $\mathrm{B}$ & 22 & 42.3 & 22 & 31.4 & 8 & 44.4 & \\
\hline $\mathrm{C}$ & 6 & 11.5 & 24 & 34.3 & 10 & 55.6 & \\
\hline
\end{tabular}

$\chi^{2}$, Chi square test MC: Monte Carlo; H, H for Kruskal Wallis test; SNP, single nucleotide polymorphism; PVT, portal vein thrombosis; CLIP score, Cancer of the Liver Italian Program Score; BCLC stage, Barcelona clinic liver cancer stage; TNM, Tumor/node/ metastasis; *, Statistically significant at $\mathrm{p}<0.05$

While no significant differences were detected regarding rs738791 genotypes or allelic frequencies when comparing
LC to HCC or healthy controls (Table 3). No statistically significant difference in the risk of HCC among all studied 
Table 5. Association between SNP rs738792 Genotype and Different Studied Parameters in HCC Group ( $\mathrm{n}=140)$.

\begin{tabular}{|c|c|c|c|c|c|c|c|}
\hline \multirow[t]{3}{*}{ Parametres } & \multicolumn{6}{|c|}{ SNP rs738792 Genotype } & \multirow[t]{3}{*}{ P-value } \\
\hline & \multicolumn{2}{|c|}{$\mathrm{TT}(\mathrm{n}=66)$} & \multicolumn{2}{|c|}{$\mathrm{TC}(\mathrm{n}=62)$} & \multicolumn{2}{|c|}{$\mathrm{CC}(\mathrm{n}=12)$} & \\
\hline & No. & $\%$ & No. & $\%$ & No. & $\%$ & \\
\hline \multicolumn{8}{|l|}{ Sex } \\
\hline Male & 52 & 78.8 & 45 & 72.6 & 10 & 83.3 & 0.597 \\
\hline Female & 14 & 21.2 & 17 & 27.4 & 2 & 16.7 & \\
\hline \multicolumn{8}{|l|}{ Age (years) } \\
\hline Range & $51.0-66.0$ & & $50.0-65.0$ & & $43.0-60.0$ & & $<0.001 *$ \\
\hline Mean $\pm \mathrm{SD}$ & $60.42 \pm 3.91$ & & $59.87 \pm 4.13$ & & $52.17 \pm 5.70$ & & \\
\hline \multicolumn{8}{|l|}{ Lymph nodes } \\
\hline No & 56 & 84.8 & 52 & 83.9 & 12 & 100 & 0.331 \\
\hline Yes & 10 & 15.2 & 10 & 16.1 & 0 & 0 & \\
\hline \multicolumn{8}{|l|}{ Metastasis } \\
\hline No & 62 & 93.9 & 62 & 100 & 12 & 100 & \\
\hline Yes & 4 & 6.1 & 0 & 0 & 0 & 0 & $\mathrm{MCp}=0.186$ \\
\hline \multicolumn{8}{|l|}{ T (Tumor stage) } \\
\hline $\mathrm{T} 1$ & 32 & 48.5 & 16 & 25.8 & 0 & 0 & \\
\hline $\mathrm{T} 2$ & 20 & 30.3 & 28 & 45.2 & 8 & 66.7 & \\
\hline $\mathrm{T} 3$ & 12 & 18.2 & 18 & 29 & 4 & 33.3 & $\mathrm{MCp}=0.002 *$ \\
\hline $\mathrm{T} 4$ & 2 & 3 & 0 & 0 & 0 & 0 & \\
\hline \multicolumn{8}{|l|}{ PVT } \\
\hline No & 50 & 75.8 & 32 & 51.6 & 0 & 0 & $<0.001 *$ \\
\hline Yes & 16 & 24.2 & 30 & 48.4 & 12 & 100 & \\
\hline $\operatorname{AFP}(\mathrm{ng} / \mathrm{ml})$ & & & & & & & 0.584 \\
\hline Median & 101.9 & & 245 & & 914.9 & & \\
\hline \multicolumn{8}{|l|}{ Focal lesion size } \\
\hline$<3 \mathrm{~cm}$ & 24 & 36.4 & 16 & 25.8 & 0 & 0 & $0.030^{*}$ \\
\hline$>3 \mathrm{~cm}$ & 42 & 63.6 & 46 & 74.2 & 12 & 100 & \\
\hline \multicolumn{8}{|c|}{ CLIP score for HCC } \\
\hline 0 & 12 & 18.2 & 0 & 0 & 0 & 0 & \\
\hline 1 & 20 & 30.3 & 4 & 6.5 & 0 & 0 & $\mathrm{MCp}$ \\
\hline 2 & 18 & 27.3 & 14 & 22.6 & 0 & 0 & \\
\hline 3 & 12 & 18.2 & 14 & 22.6 & 0 & 0 & $<0.001 *$ \\
\hline 4 & 4 & 6.1 & 16 & 25.8 & 4 & 33.3 & \\
\hline 5 & 0 & 0 & 10 & 16.1 & 6 & 50 & \\
\hline 6 & 0 & 0 & 4 & 6.5 & 2 & 16.7 & \\
\hline \multicolumn{8}{|c|}{ BCLC score for HCC } \\
\hline A & 22 & 33.3 & 2 & 3.2 & 0 & 0 & $<0.001 *$ \\
\hline $\mathrm{B}$ & 24 & 36.4 & 16 & 25.8 & 0 & 0 & \\
\hline $\mathrm{C}$ & 18 & 27.3 & 18 & 29 & 0 & 0 & \\
\hline $\mathrm{D}$ & 2 & 3 & 26 & 41.9 & 12 & 100 & \\
\hline \multicolumn{8}{|l|}{ Child score } \\
\hline A & 48 & 72.7 & 0 & 0 & 0 & 0 & $\mathrm{MCp}$ \\
\hline B & 16 & 24.2 & 36 & 58.1 & 0 & 0 & $<0.001 *$ \\
\hline $\mathrm{C}$ & 2 & 3 & 26 & 41.9 & 12 & 100 & \\
\hline
\end{tabular}

$\chi^{2}$, Chi square test; MC, Monte Carlo; H, H for Kruskal Wallis test; PVT, portal vein thrombosis; BCLC stage, Barcelona clinic liver cancer stage; TNM, Tumor/node/ metastasis; *, Statistically significant at $\mathrm{p}<0.05$.

groups regarding genotypic or allelic frequencies of rs738792 polymorphism (Table 3).

The associations between MMP-11 genotypes and different studied parameters in HCC group revealed that, no statistically significant association between the CC, CT or TT of rs738791 genotype and all of age, sex, lymph 
Table 6. Distribution of Observed SNP rs738791 and rs738792 Genotypes Frequencies and Their Consistent with Hardy-Weinberg

\begin{tabular}{|c|c|c|c|c|}
\hline Variable & Observed & Expected & $\chi^{2}$ Test & $\mathrm{p}$ value \\
\hline \multicolumn{5}{|c|}{ SNP rs738791 Genotype } \\
\hline \multicolumn{5}{|c|}{$\mathrm{HCC}(\mathrm{n}=140)$} \\
\hline $\mathrm{CC}$ & 52 & 54.1 & 0.55 & 0.458 \\
\hline $\mathrm{CT}$ & 70 & 65.9 & & \\
\hline TT & 18 & 20.1 & & \\
\hline \multicolumn{5}{|c|}{ Cirrhosis $(n=120)$} \\
\hline $\mathrm{CC}$ & 50 & 52 & 0.664 & 0.415 \\
\hline $\mathrm{CT}$ & 58 & 54 & & \\
\hline TT & 12 & 14 & & \\
\hline \multicolumn{5}{|c|}{ Controls $(n=140)$} \\
\hline $\mathrm{CC}$ & 72 & 71.4 & 0.056 & 0.812 \\
\hline $\mathrm{CT}$ & 56 & 57.1 & & \\
\hline TT & 12 & 11.4 & & \\
\hline \multicolumn{5}{|c|}{ Total $(n=400)$} \\
\hline $\mathrm{CC}$ & 174 & 176.9 & 0.421 & 0.516 \\
\hline $\mathrm{CT}$ & 184 & 178.2 & & \\
\hline TT & 42 & 44.9 & & \\
\hline \multicolumn{5}{|c|}{ SNP rs738792 Genotype } \\
\hline \multicolumn{5}{|c|}{$\mathrm{HCC}(\mathrm{n}=140)$} \\
\hline TT & 66 & 67.2 & 0.23 & 0.631 \\
\hline $\mathrm{TC}$ & 62 & 59.6 & & \\
\hline $\mathrm{CC}$ & 12 & 13.2 & & \\
\hline \multicolumn{5}{|c|}{ Cirrhosis $(n=120)$} \\
\hline TT & 60 & 58.8 & 0.272 & 0.601 \\
\hline $\mathrm{TC}$ & 48 & 50.4 & & \\
\hline $\mathrm{CC}$ & 12 & 10.8 & & \\
\hline \multicolumn{5}{|c|}{ Controls $(n=140)$} \\
\hline TT & 70 & 71.4 & 0.35 & 0.554 \\
\hline $\mathrm{TC}$ & 60 & 57.1 & & \\
\hline $\mathrm{CC}$ & 10 & 11.4 & & \\
\hline \multicolumn{5}{|c|}{ Total $(n=400)$} \\
\hline TT & 196 & 197.4 & 0.112 & 0.737 \\
\hline $\mathrm{TC}$ & 170 & 167.2 & & \\
\hline $\mathrm{CC}$ & 34 & 35.4 & & \\
\hline
\end{tabular}

If $p<0.05$ it is not consistent with Hardy-Weinberg Equilibrium (HWE)

node involvement, distant metastasis, TNM stage, AFP and tumor size. While, there was a significant association between different rs738791 genotypes and Barcelona
Matrix Metalloproteinase-11 Gene Polymorphisms in HCC

stage $(\mathrm{p}<0.001)$ as advanced Barcelona stages $(\mathrm{C}$ and D) was increased in presence of $\mathrm{T}$ allele in homozygous and heterozygous forms (CT+TT), CLIP score increased in cases with $\mathrm{T}$ allele in homozygous forms (TT) $(\mathrm{p}<$ 0.001). Also, the Child Pugh class B and C more increased in presence of $\mathrm{T}$ allele in homozygous and heterozygous forms $(\mathrm{CT}+\mathrm{TT})(\mathrm{p}<0.001)$ and PVT was more frequent in presence of $\mathrm{T}$ allele in homozygous and heterozygous forms $(\mathrm{CT}+\mathrm{TT})(\mathrm{p}=0.008)$ (Table 4$)$.

The HCC cases harboring TT, TC, and CC of rs738792 genotype showed no statistically significant association with each of sex, lymph node involvement, distant metastasis and AFP. While a significant difference was found between different genotypes and age $(\mathrm{p}<0.001)$. The presence of $\mathrm{C}$ allele of rs738792 genotype in homozygous and heterozygous forms $(\mathrm{CC}+\mathrm{CT})$ had higher risk of developing PVT $(\mathrm{p}<0.001)$, advanced tumor stage $(p=0.002)$, increased focal lesion size $>3 \mathrm{~cm}(p=0.030)$, increased CLIP score $(p<0.001)$, more advanced Barcelona stage $[D](p<0.001)$ and more advanced liver failure with Child Pugh class [B and C] $(\mathrm{p}<0.001)$ (Table 5).

Table 6 showed that the observed distribution regarding genotype frequencies of rs 738791 and rs 738792 were consistent with the expected distribution in Hardy Weinberg equilibrium.

The univariate analysis conducted on potential risk factors of $\mathrm{HCC}$, indicated that both AFP and $\mathrm{T}$ allele in homozygous and heterozygous forms $(\mathrm{CT}+\mathrm{TT})$ associated significantly $(p<0.05)$ with the increased risk of HCC against controls $(\mathrm{OR}=1.940,95 \% \mathrm{CI}: 1.514-2.486$, $\mathrm{p}<0.001 ; \mathrm{OR}=1.792,95 \%$ CI: $1.112-2.887, \mathrm{p}=0.017$ ). On multivariate analysis, all variables with $\mathrm{p}<0.05$ were included, the AFP level was independent risk factor for HCC development $(\mathrm{p}<0.001)$ with a 1.946 fold while the CT/TT genotype appeared to be non-significantly associated with risk of $\mathrm{HCC}(\mathrm{OR}=0.838,95 \% \mathrm{CI}: 0.379$ $-1.852, \mathrm{p}=0.662)$ (Table 7).

\section{Discussion}

Cirrhosis is most commonly caused by chronic hepatitis $\mathrm{C}$ infection and it is one of the major risk factors of HCC development (Berry et al., 2019). Matrix metalloproteinase-11 (MMP-11) has an important role in tumor migration and metastasis. Therefore, this study was designed to evaluate the value of two single nucleotide polymorphisms (SNPs) rs738791 and rs738792 for MMP-11 as a risk factor of HCC development among Egyptian cirrhotic patients. Two hundred sixty patients

Table 7. Univariate and Multivariate Analysis for All Studied Parameters Affecting HCC Group Versus Control

\begin{tabular}{lcccc}
\hline Variable & \multicolumn{2}{c}{ Univariate } & \multicolumn{2}{c}{ \#Multivariate } \\
& $\mathrm{p}$ & OR $(95 \% \mathrm{C} . \mathrm{I})$ & $\mathrm{P}$ & OR (95\%C.I) \\
\hline Sex (male) & 0.581 & $0.859(0.499-1.476)$ & & \\
Age (years) & 0.797 & $1.007(0.952-1.066)$ & & \\
Smoking & 0.06 & $1.610(0.979-2.646)$ & & \\
AFP & $<0.001^{*}$ & $1.940(1.514-2.486)$ & $<0.001^{*}$ & $1.946(1.516-2.499)$ \\
SNP rs738791 Genotype (CT+TT) & $0.017^{*}$ & $1.792(1.112-2.887)$ & 0.662 & $0.838(0.379-1.852)$ \\
\hline
\end{tabular}

OR, Odd 's ratio; C.I, Confidence interval; \#, All variables with $\mathrm{p}<0.05$ was included in the multivariate; *, Statistically significant at $\mathrm{p}<0.05$ 
were included in the study, 140 of them with HCC on top of $\mathrm{CHC}$ and 120 patients with post $\mathrm{CHC}$ liver cirrhosis (LC) as well as 140 subjects were enrolled in the study as healthy controls.

Diabetes mellitus (DM) in our study was significantly more prevalent among patients with LC than in those with HCC. The current data were in contrary with a study carried out by Hassan et al. (2010) who reported that diabetes appears to increase risk of HCC and this risk is correlated with a long duration of having diabetes.

In the current study, platelet count and albumin are significantly lower in both cirrhotic and HCC patients compared to control group. This was in agreement with Franca et al., (2004) who reported different theories about thrombocytopenia in chronic liver diseases. They include sequestration of platelets in spleen due to portal hypertension, decreased thrombopoietin levels, bone marrow suppression because of underlying liver disease present and auto-antibody destruction of platelets.

Dufour et al., (2000) and Harris, (2005) stated that serum level of albumin is an excellent marker of synthetic function of the liver in patients with chronic liver disease which indicates poor liver function. The most common cause for a low albumin is chronic liver failure due to presence of cirrhosis.

In the present study, a significant increase in aminotransferases (ALT and AST) was detected in HCC group more than that in cirrhosis group compared to the control group. This result was compatible with the previously stated that liver tests were elevated significantly in patients with $\mathrm{HCC}$ in comparison to chronic liver disease (Zekri et al., 2010). Also, in the present study, there was no significant difference of these parameters between cirrhosis and HCC groups. This finding was completely in agreement with the previously reported that hepatic functions cannot distinguish between HCC and cirrhosis (Silva et al., 2008). AST and ALT measure the intracellular hepatic enzymes concentration that have released into circulation and act as hepatocyte injury marker. Alkaline phosphatase, bilirubin and gamma glutamyl transpeptidase serve as markers of cholestasis and biliary function (Harris, 2005).

In our study, we found that patients with HCC had the highest level of AFP compared to cirrhotic patients and controls with a range up to $1400 \mathrm{ng} / \mathrm{ml}$. This was in agreement with a study done by Stewart in 2008 who found that AFP is increased in HCC patients and $>90 \%$ of such patients have elevated levels in the range of $400-500$ $\mathrm{ng} / \mathrm{ml}$ (Stewart, 2008). Also AFP level was significantly higher in cirrhotic patients in comparison with controls in our study, which agreed with Wang et al., (2005) and Tai et al., (2009) who reported in their study raised levels of AFP in cirrhotic patients without HCC. Di Bisceglie et al., (2005) observed that AFP production is triggered by presence of inflammation, occurrence of hepatocellular injury and necrosis, possibly as a result of increased turnover of hepatocyte.

Also, Mousa et al., (2012) reported that elevated levels of serum AFP may be a result of altered hepatocyte-hepatocyte interaction which is associated with a loss of normally present architectural arrangements rather than active regeneration or necrosis. Chaiteerakij et al., (2015) reported that, serum level of AFP is considered as early diagnostic and prognostic biomarker for $\mathrm{HCC}$, and it is an independent risk predictor of pathological grade, disease progression, patient survival and tumor recurrence (Bai et al., 2017).

Moreover the MMP-1 polymorphism in the present study, no significant difference among studied groups was found as regards the SNP rs738791 genotypes. While the CT and TT genotypes combination was more frequent in HCC group compared to LC and control. Also, a higher frequency of T allele was detected in HCC patients $(37.9 \%)$ compared to cirrhotic patients $(34.2 \%)$ and healthy controls $(28.6 \%)$ without statistical significant difference. No significant difference was observed among studied groups as regards SNP rs738792 genotype (TT, $\mathrm{TC}$ or $\mathrm{CC}$ ), allele distributions ( $\mathrm{T}$ or $\mathrm{C}$ ) or combination of TC + CC genotypes.

The risk of hepatocellular carcinoma among different genotypes of rs 738791 (TT and CT genotype) was significantly increased by 2.077 fold and 1.731 fold respectively when compared to healthy control. Additionally, the patients carrying heterozygous (CT+TT) genotype and $\mathrm{T}$ allele had a significant increased 1.791 fold and 1.523 fold respectively when compared to controls. While no significant differences were detected regarding rs738791 genotypes or allelic frequencies when comparing LC to HCC or healthy controls. These findings suggest that different MMP-11 polymorphisms may play different roles in development of cancer.

In contrast, no statistically significant difference among all studied groups regarding genotypic or allelic frequencies of rs738792 polymorphism was found. These results were compatible with the previously reported study Wang et al., (2018) as they found that there was no significant difference as regards $\mathrm{HCC}$ patients with the rs 738792 polymorphisms when compared with healthy controls.

The associations between MMP-11 genotypes and different studied parameters in HCC group revealed that, no statistically significant association between the CC, CT or TT of rs738791 genotype and all of age, sex, lymph node involvement, distant metastasis, TNM stage, AFP and tumor size. These results were also obtained by Wang et al., (2018) who revealed that no significant associations between AFP serum level in HCC patients and genotypes of any MMP-11 SNPs.

There was a significant association between different rs738791 genotypes and Barcelona stage as advanced Barcelona stages (C and D) was increased in presence of T allele in homozygous and heterozygous forms (CT+TT), CLIP score increased in cases with T allele in homozygous forms (TT). Also, the Child Pugh class B and C more increased in presence of $\mathrm{T}$ allele in homozygous and heterozygous forms $(\mathrm{CT}+\mathrm{TT})$ and PVT was more frequent in presence of $\mathrm{T}$ allele in homozygous and heterozygous forms $(\mathrm{CT}+\mathrm{TT})$. MMP-1 high expression proved to be a risk factor for tumor recurrence and independent molecular marker of prognosis in HCC and may become a novel target in the strategies for the prediction of tumor progression and prognosis of this disease (Yassen et al., 
2013).

On the other hand, the HCC cases harboring TT, TC, and CC of rs738792 genotype showed no statistically significant association with each of sex, lymph node involvement, distant metastasis and AFP. While significant difference was found between different genotypes and age. The presence of $\mathrm{C}$ allele of rs 738792 genotype in homozygous and heterozygous forms $(\mathrm{CC}+\mathrm{CT}) \mathrm{had}$ higher risk of developing PVT, advanced tumor stage, increased focal lesion size $>3 \mathrm{~cm}$, increased CLIP score, more advanced Barcelona stage [D] and more advanced liver failure with Child Pugh class [B and C].These results agreed with Wang et al. (2018) who reported that no significant associations between the levels of HCC clinical pathologic markers and genotypes of any MMP-11 SNPs.

The current study revealed that the observed distribution regarding genotype frequencies of rs 738791 and rs738792 and Hardy-Weinberg, the distribution was consistent with the expected distribution in Hardy Weinberg equilibrium.

The univariate analysis conducted on potential risk factors of $\mathrm{HCC}$ in our work indicated that both AFP and T allele of SNP rs 738791 in homozygous and heterozygous forms $(\mathrm{CT}+\mathrm{TT})$ was associated significantly $(\mathrm{p}<0.05)$ with the increased risk of $\mathrm{HCC}$ against controls $(\mathrm{OR}=1.940$, 95\% CI: $1.514-2.486, \mathrm{p}<0.001 ; \mathrm{OR}=1.792,95 \% \mathrm{CI}$ : $1.112-2.887, \mathrm{p}<0.017$ ). Also, Wang et al., 2018 reported that after potential confounders adjustment, subjects with combined CT and TT of MMP-11 rs738791 polymorphism had a 1.389 -fold- ( $\% 95$ CI: $1.004-1.921 ; \mathrm{p}<0.05$ ) higher risk of $\mathrm{HCC}$ development compared to those with homozygotes $\mathrm{C} / \mathrm{C}$.

In conclusion, we found that the genetic polymorphisms in the Matrix metalloproteinases-11 (MMP-11) gene: rs738791, but not rs 738792 was significantly associated with risk of HCC development in Egyptian patients with chronic HCV infection. Carriers of the $\mathrm{CT}+\mathrm{TT}$ allele of the rs 738791 variant were at greater risk of HCC compared with wild-type (CC) carriers. Furthermore, the presence of $\mathrm{C}$ allele of rs 738792 genotype in homozygous and heterozygous forms $(\mathrm{CC}+\mathrm{CT})$ had higher risk of developing PVT, increased tumor size, advanced tumor stages, higher CLIP score, advanced Barcelona stage and more advanced liver cell failure. Future large scale studies are recommended to clarify the role of other genetic variation in the $M M P$ - 11 gene that may help in understanding the pathogenesis of $\mathrm{HCC}$ and to be targeting for therapy.

\section{Acknowledgements}

Deep thanks to all members of National liver Institute for their support

\section{Conflict of interest}

No conflict of interest to be declared.

All authors share the clinical, pathological, and statistical assessment prospects of the manuscript added to the writing.

\section{References}

Bai DS, Zhang C, Chen P, Jin SJ, Jiang GQ (2017): The prognostic correlation of AFP level at diagnosis with pathological grade, progression, and survival of patients with hepatocellular carcinoma. Sci Rep, 7, 12870.

Berry E, Avraham Y, Mechoulam R, et al (2019):Treatment of hepatic encephalopathy and liver cirrhosis; U.S. Patent No. 10, pp 166-202.

Bi Q, Tang S, Xia L, et al (2012): Ectopic expression of MiR-125a inhibits the proliferation and metastasis of hepatocellular carcinoma by targeting MMP11 and VEGF. PLoS One, 7, e40169.

Chaiteerakij R, Zhang X, Addissie BD, et al (2015). Combinations of biomarkers and Milan criteria for predicting hepatocellular carcinoma recurrence after liver transplantation. Liver Transplantation, 21, 599-606.

Di Bisceglie AM (2005): Pretransplant treatments for hepatocellular carcinoma: Do they improve outcomes? Liver Transpl, 11, 10-3.

Dufour DR, Lott JA, Nolte FS, et al (2000). Diagnosis and monitoring of hepatic injury. II. Recommendations for use of laboratory tests in screening, diagnosis, and monitoring. Clin Chem, 46, 2050-68.

European Association for the Study of the Liver (2018): EASL Clinical Practice Guidelines: Management of hepatocellular carcinoma. J Hepatol, 69, 182-236.

França AV, Elias Junior J, Lima BL, Martinelli AL, Carrilho FJ (2004). Diagnosis, staging and treatment of hepatocellular carcinoma. Braz J Med Biol Res, 37, 1689-705.

Geervliet E, Bansal R (2020). Matrix metalloproteinases as potential biomarkers and therapeutic targets in liver diseases. Cells, 9, 1212.

Gupta H, Youn GS, Shin MJ, Suk KT (2019). Role of gut microbiota in hepatocarcinogenesis. Microorganisms, 7, 121.

Harris EH (2005). Elevated liver function tests in type 2 diabetes. Clin Diabetes, 23, 115-9.

Hassan MM, Curley SA, Li D, et al (2010). Association of diabetes duration and diabetes treatment with the risk of hepatocellular carcinoma. Cancer, 116, 1938-46.

Josep QU, Rodríguez F, Gregori J (2019). Primers and methods for detecting human hepatitis $\mathrm{C}$ virus (HCV) variants in an isolated sample; U.S. Patent Application No. 10/202,659.

Kessenbrock K, Plaks V, Werb Z(2010). Matrix metalloproteinases: regulators of the tumor microenvironment. Cell, 141, 52-67.

Koleck TA, Bender CM, Clark BZ, et al (2017). An exploratory study of host polymorphisms in genes that clinically characterize breast cancer tumors and pretreatment cognitive performance in breast cancer survivors. Breast Cancer Targets Therapy, 9, 95-110.

Lin CW, Yang SF, Chuang CY, Lin HP, Hsin CH (2015). Association of matrix metalloproteinase-11 polymorphisms with susceptibility and clinicopathologic characteristics for oral squamous cell carcinoma. Head Neck, 37, 1425- 31.

Mousa N, Gad Y, Abdel-Aziz A, Abd-Elaal I (2012). Increased $\alpha$-fetoprotein predicts steatosis among patients with chronic hepatitis $\mathrm{C}$ genotype 4. Int $J$ Hepatol, 2012, Article ID 636392, 5 pages.

Pugh RN, Murray-Lyon IM, Dawson JL, Pietroni MC, Williams R (1973). Transection of the esophagus for bleeding esophageal varices. Br J Surg, 60, 646-9.

Rouyer N, Wolf C, Chenard MP, Rio MC, Chambon P, et al (1994). Stromelysin-3 gene expression in human cancer: an overview. Invasion Metastasis, 14, 269-75.

Silva M, Moya A, Berenguer M, et al (2008). Expanded criteria for liver transplantation in patients with cirrhosis and hepatocellular carcinoma. Liver Transplantation, 14, 
1449-60.

Stewart MW, Rosenfeld PJ (2008). Predicted biological activity of intravitreal VEGF Trap. Br J Ophthalmol, 92, 667-8.

Tai WC, Hu TH, Wang JH, et al (2009). Clinical implications of alpha-fetoprotein in chronic hepatitis C. J Formos Med Assoc, 108, 210-8.

Tecnologies L (2013). PureLink ${ }^{\circledR}$ Genomic DNA Kit: for purification of genomic DNA. Document Part, (25-1012).

Wang B, Hsu CJ, Lee HL, et al (2018). Impact of matrix metalloproteinase-11 gene polymorphisms upon the development and progression of hepatocellular carcinoma; Int J Med Sci, 15, 653.

Wang Y, Mao XO, Xie L (2005). VEGF overexpression delays neurodegeneration and prolongs survival in ALS mice. The Journal of neuroscience: the official journal of the Society for Neuroscience, 27, 304-7.

Yassen MEAFAA, Hammam OAI, Sarhan HKAAM (2013). Expression of matrix metalloproteinase 1 (MMP1) in hepato cellular carcinoma (HCC): Immunohistochemical and biochemical studies. Egypt J Hospital Med, 51, 289-99.

Zekri ARN, El-Din HMA, Bahnassy AA, et al (2010). Serum levels of soluble Fas, soluble tumor necrosis factor-receptor II, interleukin-2 receptor and interleukin-8 as early predictors of hepatocellular carcinoma in Egyptian patients with hepatitis $\mathrm{C}$ virus genotype-4. Comparative Hepatol, 9, 1.

Zhang X, Huang S, Guo J, et al (2016). Insights into the distinct roles of MMP-11 in tumor biology and future therapeutics (Review). Int J Oncol, 48, 1783-93.

Zhu K, Dai Z, Zhou J (2013). Biomarkers for hepatocellular carcinoma: progression in early diagnosis, prognosis, and personalized therapy. Biomarker Res, 1, 10.

\section{๑๐ब}

This work is licensed under a Creative Commons AttributionNon Commercial 4.0 International License. 